\title{
Use of fusion transcription factors to reprogram cellulase transcription and enable efficient cellulase production in Trichoderma reesei
}

Fangzhong Wang ${ }^{1,2,3}$, Ruiqin Zhang ${ }^{1}$, Lijuan Han' ${ }^{1}$, Wei Guo ${ }^{1}$, Zhiqiang Du' ${ }^{1}$ Kangle Niu', Yucui Liu', Chunjiang $\mathrm{Jia}^{3}$ and Xu Fang ${ }^{1^{*}}$ (D)

\begin{abstract}
Background: Trichoderma reesei is widely used for cellulase production and accepted as an example for cellulase research. Cre1-mediated carbon catabolite repression (CCR) can significantly inhibit the transcription of cellulase genes during cellulase fermentation in T. reesei. Early efforts have been undertaken to modify Cre 1 for the release of CCR; however, this approach leads to arrested hyphal growth and decreased biomass accumulation, which negatively affects cellulase production.

Results: In this study, novel fusion transcription factors (fTFs) were designed to release or attenuate CCR inhibition in cellulase transcription, while Cre1 was left intact to maintain normal hyphal growth. Four designed fTFs were introduced into the T. reesei genome, which generated several transformants, named Kuace3, Kuclr2, Kuace2, and Kuxyr1. No obvious differences in growth were observed between the parent and transformant strains. However, the transcription levels of cel7a, a major cellulase gene, were significantly elevated in all the transformants, particularly in Kuace 2 and Kuxyr1, when grown on lactose as a carbon source. This suggested that CCR inhibition was released or attenuated in the transformant strains. The growth of Kuace 2 and Kuxyr 1 was approximately equivalent to that of the parent strain in fed-batch fermentation process. However, we observed a 3.2- and 2.1-fold increase in the $p$ NPCase titers of the Kuace 2 and Kuxyr1 strains, respectively, compared with that of the parent strain. Moreover, we observed a 6.1- and 3.9-fold increase in the pNPCase titers of the Kuace2 and Kuxyr1 strains, respectively, compared with that of $\Delta$ crel strain.
\end{abstract}

Conclusions: A new strategy based on fTFs was successfully established in T. reesei to improve cellulase titers without impairing fungal growth. This study will be valuable for lignocellulosic biorefining and for guiding the development of engineering strategies for producing other important biochemical compounds in fungal species.

Keywords: Fusion transcription factors, Carbon catabolite repression, Biomass, Cellulase, Trichoderma reesei, Cre1

\footnotetext{
*Correspondence: fangxu@sdu.edu.cn

${ }^{1}$ State Key Laboratory of Microbial Technology, Shandong University, Qingdao 266237, China

Full list of author information is available at the end of the article
} 


\section{Background}

Despite being the most abundant natural material, lignocellulose is a considerably under-utilized biomass $[1,2]$. Lignocellulosic biorefining processes producing biofuels have attracted considerable attention in the past few decades owing to the global energy crisis caused by the extensive depletion of fossil fuels. In addition, large quantities of high-value chemical intermediates can be generated during the process [1-4]. One of the most important steps in lignocellulosic biorefining is the use of cellulases to release fermentable sugar from lignocellulose [5, 6]. However, a considerable limiting factor in industrial lignocellulosic biorefining is the high cost of the vast amounts of cellulases that are required for the degradation of lignocellulose (40- to 100-fold higher than that required for starch hydrolysis) [7-9].

One promising strategy for decreasing enzyme costs is to increase cellulase production by filamentous fungi [10]. However, the presence of D-glucose or D-xylose during cellulase fermentation triggers carbon catabolite repression (CCR), which significantly decreases cellulase transcription in filamentous fungi [11]. Therefore, the release or attenuation of CCR is a prerequisite for high-yield cellulase production [12-14]. In filamentous fungi, CCR is mediated by the conserved transcription factor Cre1. Long et al. [15] reported that the cellulase activity of $\Delta c r e 1$ strain was 1.45 -fold higher than that of its parent strain of Trichoderma orientalis EU722. Furthermore, Sun and Glass [16] demonstrated that the knockout of cre1 in Neurospora crassa improved the expression of most cellulases and increased endocellulase activity by approximately $50 \%$. Therefore, the deletion of cre1 is widely accepted as an effective strategy for releasing CCR. However, several studies have revealed that cre1 deletion impairs filamentous fungal growth, which leads to decreased final product titers. For example, the deletion of cre 1 in $T$. reesei or its replacement with a cre1 mutant inhibited hyphal growth and affected final biomass accumulation, which led to decreased cellulase production $[11,17,18]$. In addition, $\Delta c r e 1$ strain exhibited slower growth than its parent strain [16]. However, Rassinger et al. found that Cre1-96, a truncated from of Cre1, could bind to the cellulase promoter regions in $T$. reesei Rut-C30 and contributed to a partial release from CCR [19]. Furthermore, the constitutive expression of cre1-96 could improve cellulase activity without impairing strain growth [20]. However, its deletion significantly decreased cellulase activity and resulted in serious growth defects [20]. These observations suggested that the truncation caused Cre1-96 to become an activator of cellulase expression rather than a repressor and that the functions of Cre1 exhibited a degree of plasticity. However, a structure-based approach for the rational design of Cre1 mutants is not feasible owing to the absence of crystal structure information.

The construction of synthetic transcription factors involves the fusion of transcription factor zinc finger domains with the activation domains of well-studied activators or repressors. They have demonstrated significant potential as a tool for creating phenotypic alterations in yeast and mammalian cells [21-24]. Replacement of the activation domain determines the specific role of a synthetic transcription factor [21]. Therefore, this strategy may provide a solution for the rational design of Cre1based fusion transcription factors that can release or attenuate CCR in filamentous fungi. As previously mentioned, the deletion of cre1 led to serious growth defects. Furthermore, Cre1 is required, albeit indirectly, for the positive regulation of key genes involved in fundamental life processes, such as energy biosynthesis and general metabolism [18]. Therefore, we proposed that the construction of fusion transcription factors based on Cre1 is an appropriate alternative for releasing or attenuating CCR in filamentous fungi. This would relieve the repressive effect of Cre1 on cellulase production; however, the maintenance of fungal growth will not be affected.

The industrial production of cellulase involves the fermentation of filamentous fungi, such as $T$. reesei, N. crassa, and Penicillium oxalicum [25-28]. Of these strains, T. reesei has the highest potential for the overproduction and secretion of cellulases [29, 30]. For example, the cellulase volumetric productivity rate of T. reesei CL847 can reach levels of $243 \mathrm{FPU} / \mathrm{L} / \mathrm{h}$ in a 3000-L fermenter using lactose as a carbon source [31]. Furthermore, the cellulases currently required for global cellulosic ethanol production are predominantly derived from $T$. reesei [32].

The cellulase transcriptional regulatory network in $T$. reesei has been extensively explored in the last few years, and the functions of cellulase transcriptional repressors and activators in $T$. reesei have been comprehensively characterized [2]. Cre1 and Ace1 are cellulase transcriptional repressors that directly bind to the promoter regions of cellulase genes [2], and Ace3, Clr2, Ace2, and Xyr1 are transcriptional activators that enhance the transcriptional expression of cellulase genes [2,33]. Therefore, we proposed that the $T$. reesei is a suitable system for screening novel synthetic transcription factors owing to their ability to release or attenuate CCR while retaining intact Cre1 to allow effective fungal growth. Such efforts will also accelerate the industrialization of biorefining processes.

Here, we proposed a novel strategy to alleviate CCR in $T$. reesei that was based on the construction of fusion transcription factors (fTFs). Four fTFs were constructed and integrated into the $T$. reesei genome and 
the phenotypic characteristics of the transformants were investigated in detail. Furthermore, we examined the transcription levels of the cellulase transcription factors and the major cellulase gene cel7a in the fTF transformants to confirm the effectiveness of our strategy for activating cellulase transcription. In addition, the cellulase titers of the transformants were compared with those of the parent and $\Delta c r e 1$ strains using fed-batch culture in 3-L fermenters. This approach could also be employed in other fungal strains to release CCR while maintaining normal strain growth.

\section{Results}

\section{Construction of fTFs}

The fTFs were designed to include four parts: the activation domain of a cellulase activator protein (Ace3, Clr2, Ace2, or Xyr1), the zinc finger regions of Cre1 and Ace1, and a peptide used to link each domain (Fig. 1a). These fTFs were named Sace3, Sclr2, Sace2, and Sxyr1. Each fTF was under the control of the T. reesei cre1 promoter and the Aspergillus nidulans trpC terminator (Additional file 1: Figure S1A). The zinc finger regions of the fTFs are believed to alleviate Cre1-and Ace1-mediated repression by competing for the Cre1- and Ace1-binding sites (Fig. 1b).

The constructed fTF cassettes were introduced into a $T$. reesei $\Delta t k u 70$ strain to obtain the transformants, which were named Kuace3, Kuclr2, Kuace2, and Kuxyr1. Successful insertion of the cassettes was confirmed by Southern blotting (Additional file 1: Figure S1B, C). In addition, we constructed a control $T$. reesei $\Delta c r e 1$ strain (Additional file 2: Figure S2).

\section{Phenotypic characterization of the transformants}

Equivalent amounts of mycelia from the parent strain and transformants were inoculated on plates containing glucose, lactose, wheat bran, or potato dextrose agar (PDA). Phenotypic analysis of each strain was performed after incubating the plates for 6 days at $30{ }^{\circ} \mathrm{C}$. Figure 2 shows the morphological changes observed in each strain on various plates. On plates containing lactose as a carbon source, the Kuclr2, Kuace2, and T. reesei $\Delta$ cre1 colonies became denser; in addition, the Kuace2 exhibited an annular distribution. Furthermore, significant differences in pigment production by the Kuclr2, Kuace2, $\Delta c r e 1$, and parent strains were observed on PDA and wheat brancontaining plates. On plates containing glucose, lactose, wheat bran, and PDA, the T. reesei $\triangle$ crel colonies were significantly smaller in diameter than those of the parent strain. In contrast, the colony diameters of Kuace3, Kuclr2, Kuace2, and Kuxyr1 were equivalent to those of the parent strain on PDA and wheat bran-containing plates (Additional file 3: Figure S3). We observed a slight decrease in the Kuclr2 and Kuace2 colony diameters when cultured on lactose-containing plates. Although the Kuace 2 and Kuxyr1 colonies exhibited smaller diameters when cultured on plates containing glucose, their diameters were still higher than those of $T$. reesei $\Delta$ cre1. Microscopic observation showed no hyphal differences among the Kuace3, Kuclr2, Kuace2, Kuxyr1, and parent strains. However, the hyphae of T. reesei $\Delta c r e 1$ were notably shorter and thicker (Additional file 4: Figure S4). Next, the sporulation ability of all the strains was tested. The number of spores produced by all the transformants was found to drastically reduce. In particular, compared with the parent strain, the $\Delta c r e 1$ strain produced five or sixfold lower numbers of spores on plates containing PDA or wheat bran, respectively (Additional file 5: Figure S5). Based on these observations, we concluded that the fTFs caused fewer morphological changes in T. reesei in comparison with cre1 deletion. There were no apparent differences in hyphal growth between the parent and
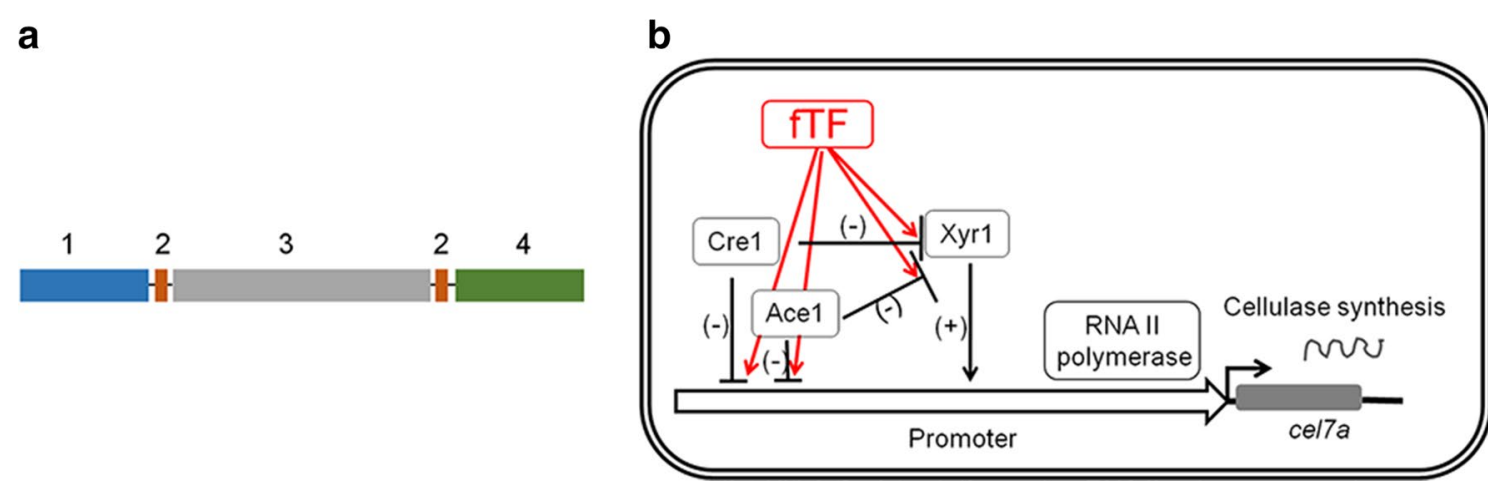

Fig. 1 Schematic diagram and hypothetical working mechanism of fTFs in T. reesei. a 1: the Cre1 zinc finger region; 2: peptide linker: GSGGSGTS; 3: the activation domain of Ace3, Clr2, Ace2, or Xyr1; 4: the Ace 1 zinc finger region. $\mathbf{b}$ - represents repression; + represents activation, and cel7a represents cellobiohydrolase 1 


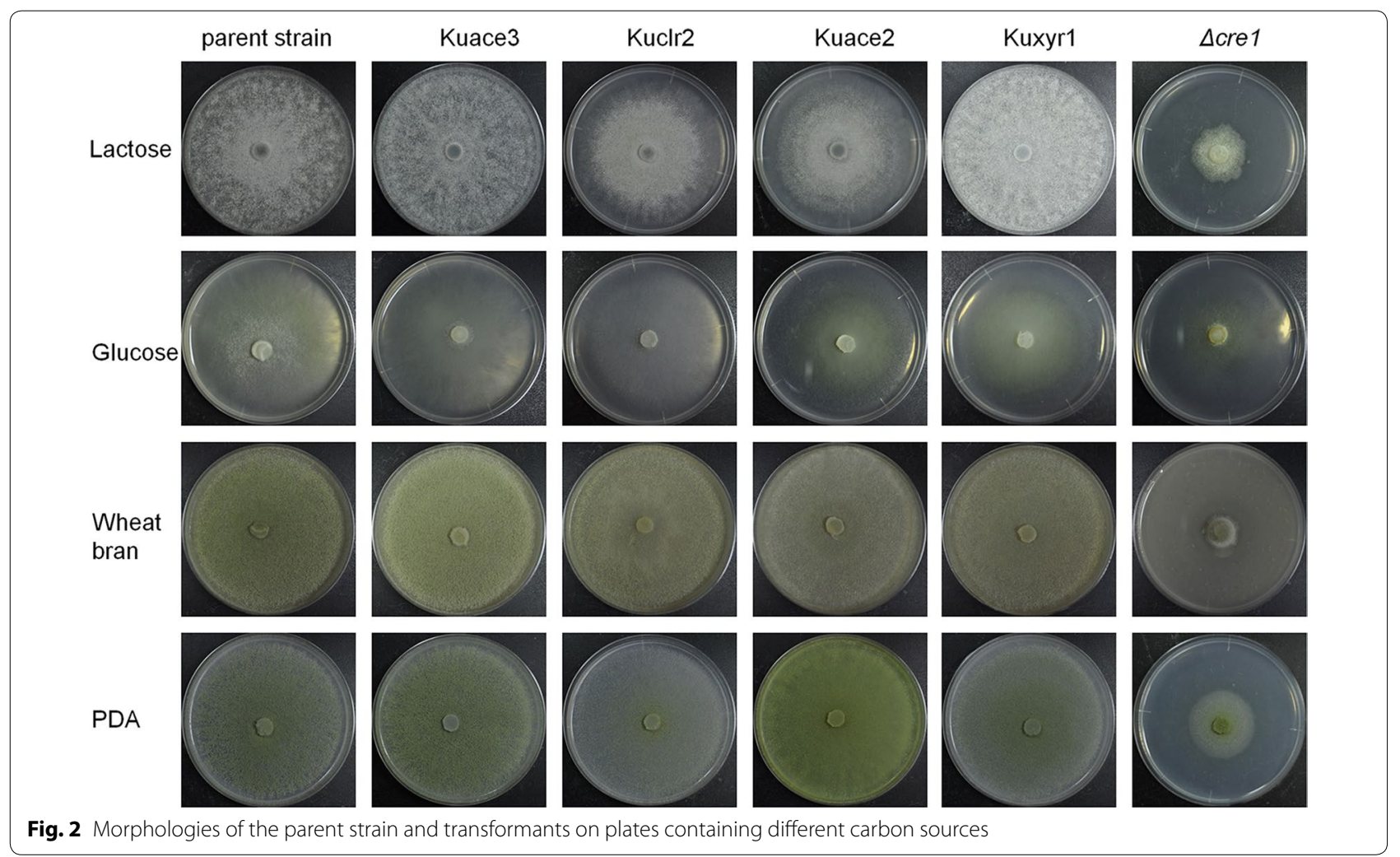

fTF-containing transformant strains, with the exception of Kuace 2 and Kuxyr1 cultured on glucose-containing plates (Fig. 2 and Additional file 3: Figure S3).

\section{Transcriptional levels of $x y r 1$ and cel7a following the homologous integration of fTFs into the $T$. reesei genome}

The growth of Kuace3, Kuclr2, Kuace2, or Kuxyr1 was not obviously impaired on plates containing lactose. Lactose is considered to be an economically feasible carbon source for inducing cellulase expression in T. reesei [3437]. Therefore, we decided to use lactose as the carbon source for all our subsequent investigations in this study.

Next, we used qRT-PCR to investigate the expression levels of genes encoding cellulase transcription factors in the Kuace3, Kuclr2, Kuace2, Kuxyr1, and parent strains following their incubation in media containing $2 \%$ lactose for $1,6,8$, and $12 \mathrm{~h}$. The transcriptional levels of most cellulase transcription factors were unaltered (data not shown). However, $x y r 1$, which encodes the main cellulase activator in T. reesei [11], was significantly upregulated in the Kuace3, Kuclr2, Kuace2, and Kuxyr1 strains (Fig. 3).

When $T$. reesei Rut-C30 was cultured in a two-stage system with lactose as a carbon source and a controlled $\mathrm{pH}$ of 4.8, the major secreted cellulase was cellobiohydrolase 1 , which can account for approximately $50 \%$ of

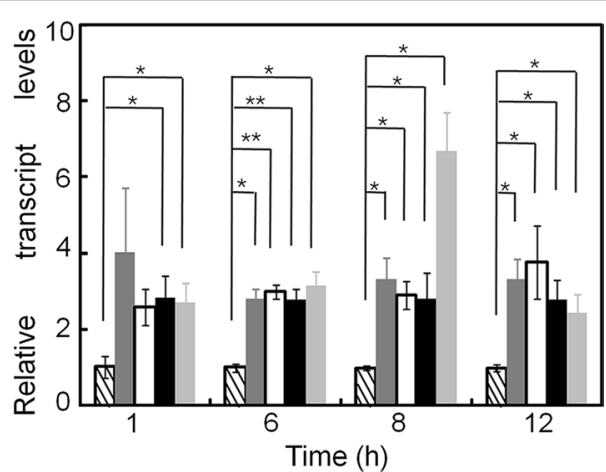

Fig. $3 x y r 1$ transcriptional levels in the parent strain and fTF-containing transformants. The left slash represents the parent strain, dark gray represents Kuace3, white represents Kuclr2, black represents Kuace2, and light gray represents Kuxyr1. The transcriptional level of $x y r 1$ was normalized to that of actin (data presented are mean \pm SEM; ${ }^{*} p<0.05,{ }^{* *} p<0.01, n=3$; two-tailed Student's $t$ tests)

the total extracellular protein [38]. Cellobiohydrolase 1 is encoded by the cel7a gene. Therefore, we examined the transcriptional level of cel7a in all our strains. As shown in Fig. 4, the transcriptional levels of cel7a in Kuace3, Kuclr2, Kuace2, and Kuxyr1 were all significantly upregulated at 6 (Fig. 4b), 8 (Fig. 4c), and 12 h (Fig. 4d) after 

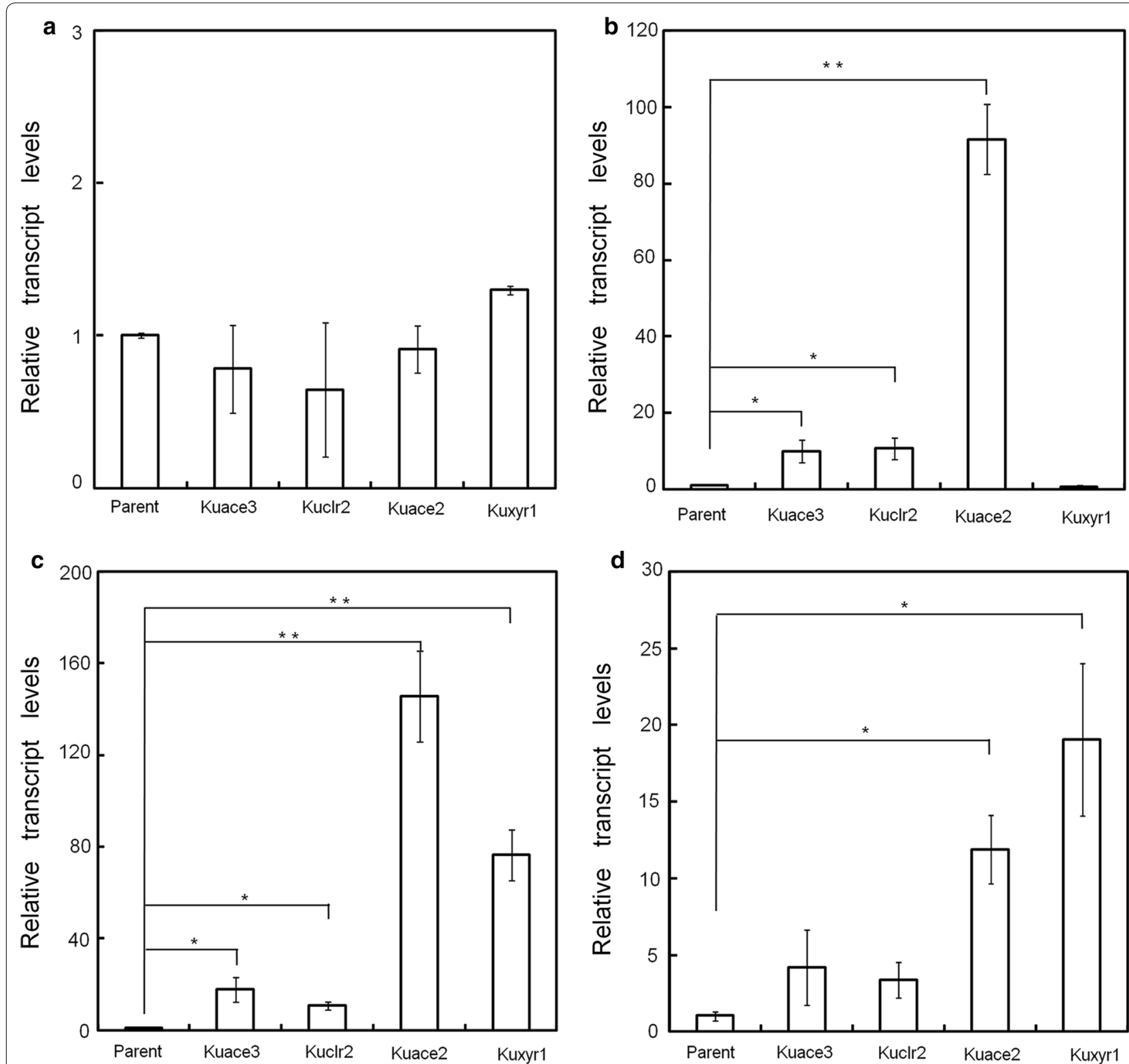

Fig. 4 Transcriptional levels of cel7a in the parent strain and fTF-containing transformants. a Cultivation for 1 h; $\mathbf{b}$ cultivation for 6 h; c cultivation for $8 \mathrm{~h}$; $\mathbf{d}$ cultivation for $12 \mathrm{~h}$. The transcriptional level of the cel7a gene was normalized to that of actin (data presented are mean $\pm \mathrm{SEM} ;{ }^{*} p<0.05$, ${ }^{* *} p<0.01,{ }^{* * *} p<0.001, n=3$; two-tailed Student's $t$ tests)

transferring to media containing lactose. The expression of cel7a in the Kuace2 strain was upregulated by approximately 100 -fold at 6 or $8 \mathrm{~h}$ following its transfer to lactose-containing media. Furthermore, the expression of cel7a was upregulated by approximately 77 -fold in the Kuxyr1 strain at $8 \mathrm{~h}$ and approximately tenfold in the Kuace 2 and Kuxyr1 strains at $12 \mathrm{~h}$. Although the expression of cel7a was significantly enhanced in the Kuace3 or Kuclr2 strains at 6, 8, or $12 \mathrm{~h}$, the extent of upregulation was much lower than that of Kuace2 and Kuxyr1.
There were no significant changes in the transcriptional levels of the fTFs (Sace3, Sclr2, Sace2, and Sxyr1) among the transformants (Additional file 6: Figure S6). Conclusively, although all fTFs were able to significantly improve the transcription of both $x y r 1$ and cel7a in T. reesei, the augmentations exhibited by strains containing Sace 2 or Sxyr1 were much higher than those containing Sace3 or Sclr2. Therefore, we concluded that the fTFs must have different functions that led to these observed changes in expression levels. 
Determination of cellulase activity in shake-flask cultures As Kuace2 and Kuxyr1 exhibited the highest cel7a transcription levels (Fig. 4), we compared the $p$ NPCase (cellobiohydrolase 1) activities of Kuace2, Kuxyr1, and the parent strain. For each of these strains, $p$ NPCase activities were determined, and the results were expressed as enzyme unit per dry mycelium weight. Although the $p$ NPCase activities in Kuace2 and Kuxyr1 were significantly increased compared with that of the parent strain $(p<0.05, n=3)$ (Fig. 5a), the biomass accumulations of these strains were not significantly altered (Fig. 5b). These findings indicate that both Sace 2 and Sxyr 1 are able to significantly enhance $p$ NPCase activities in T. ree$s e i$ without affecting biomass accumulation.

\section{Cellulase production in 3-L fermenters}

As cellobiohydrolase 1 expression in the Kuace 2 or Kuxyr1 strains was significantly elevated in shake-flask cultures (Figs. 4 and 5), we scaled up the fed-batch cultures to assess cellobiohydrolase 1 production by the Kuace2, Kuxyr1, $\Delta$ cre1, and parent strains in 3-L fermenters. Supplementary medium containing lactose as a carbon source and $\left(\mathrm{NH}_{4}\right)_{2} \mathrm{SO}_{4}$ as a nitrogen source was added after $66 \mathrm{~h}$ of cultivation. The intracellular protein concentration was measured to determine the biomass concentration in each culture $[39,40]$. In addition, the $\mathrm{pH}$ was controlled at 4.0-5.0, and no significant changes in $\mathrm{pH}$ values were observed during the whole fermentation processes for any of the strains (data not shown).

As shown in Fig. 6, the $p$ NPCase activities were significantly increased in the Kuace 2 and Kuxyr1 strains compared with those in the parent and $\Delta c r e 1$ strains after feeding was complete $(p<0.05, n=3)$. The biomass accumulation of the Kuace2, Kuxyr1, and parent strains significantly increased after $108 \mathrm{~h}$ of cultivation and was noted to be maximum at $180 \mathrm{~h}$. In contrast, the biomass concentration of the $\Delta c r e 1$ strain slowly increased throughout its cultivation (Fig. 6b). We observed no significant differences in biomass accumulation among the Kuace2, Kuxyr1, and parent strains. However, the $\Delta$ cre 1 strain biomass was approximately one-quarter or one-third of the concentration reached by the Kuace 2 or Kuxyr 1 strains, respectively. Furthermore, the $p$ NPCase titers of Kuace 2 and Kuxyr1 were strongly increased following the completion of feeding compared with those of the parent and $\Delta c r e 1$ strains (Fig. 6a). After $180 \mathrm{~h}$ of fermentation, the $p$ NPCase titers of Kuace2 and Kuxyr1 were 3.2- and 2.1-fold higher, respectively, than that of the parent strain

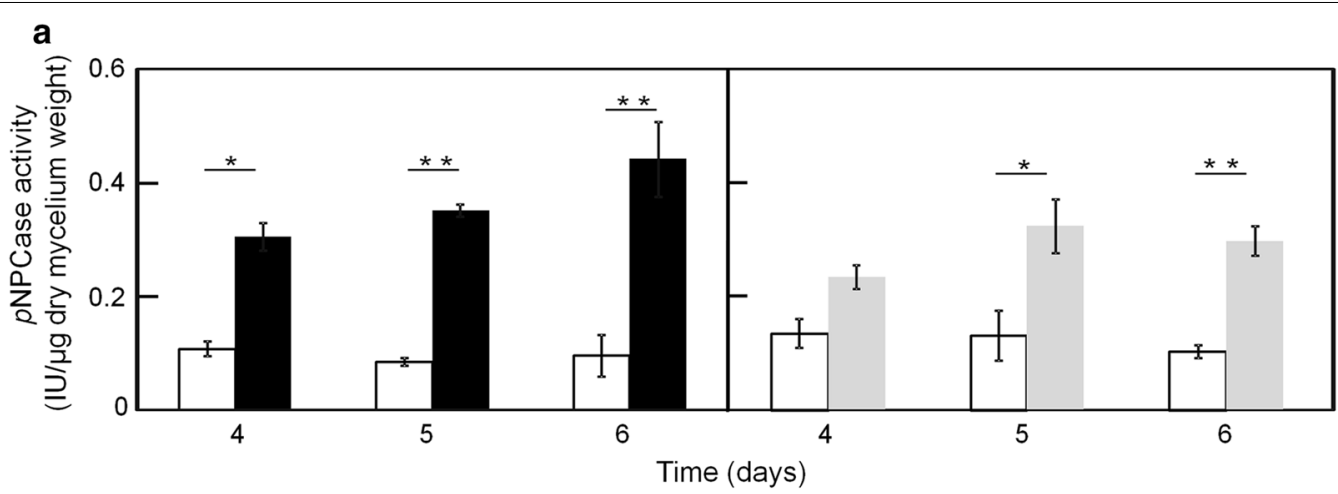

b

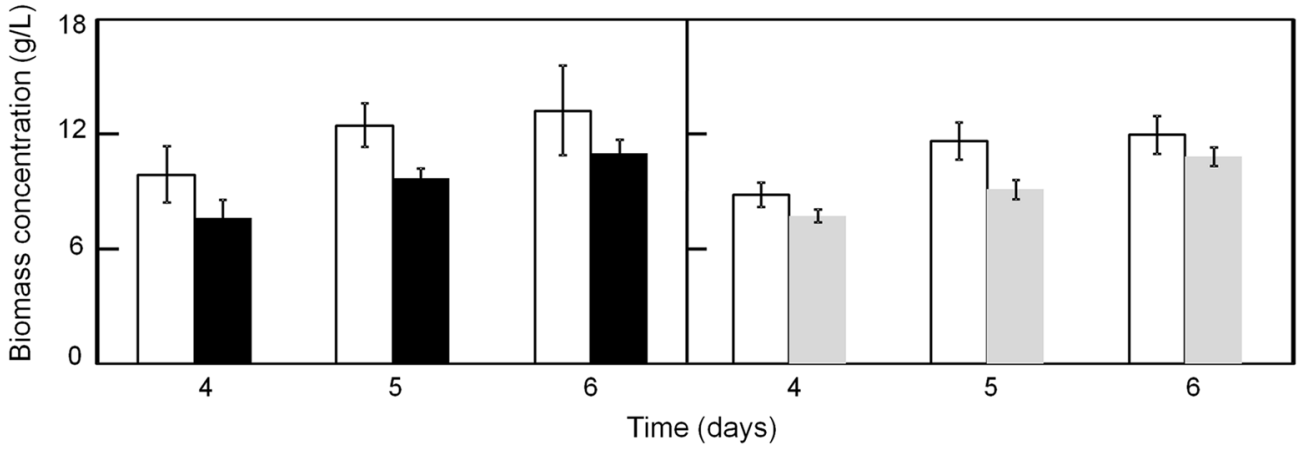

Fig. 5 Comparison of $p N P C a s e$ activity and biomass concentration among strains in shake-flask cultures. The white symbol represents the parent strain, black represents Kuace2, and gray represents Kuxyr1. a $p$ NPCase activity; b biomass concentration (data presented are mean $\pm S E M ;{ }^{*} p<0.05$, ${ }^{* *} p<0.01, n=3$; two-tailed Student's $t$ tests) 

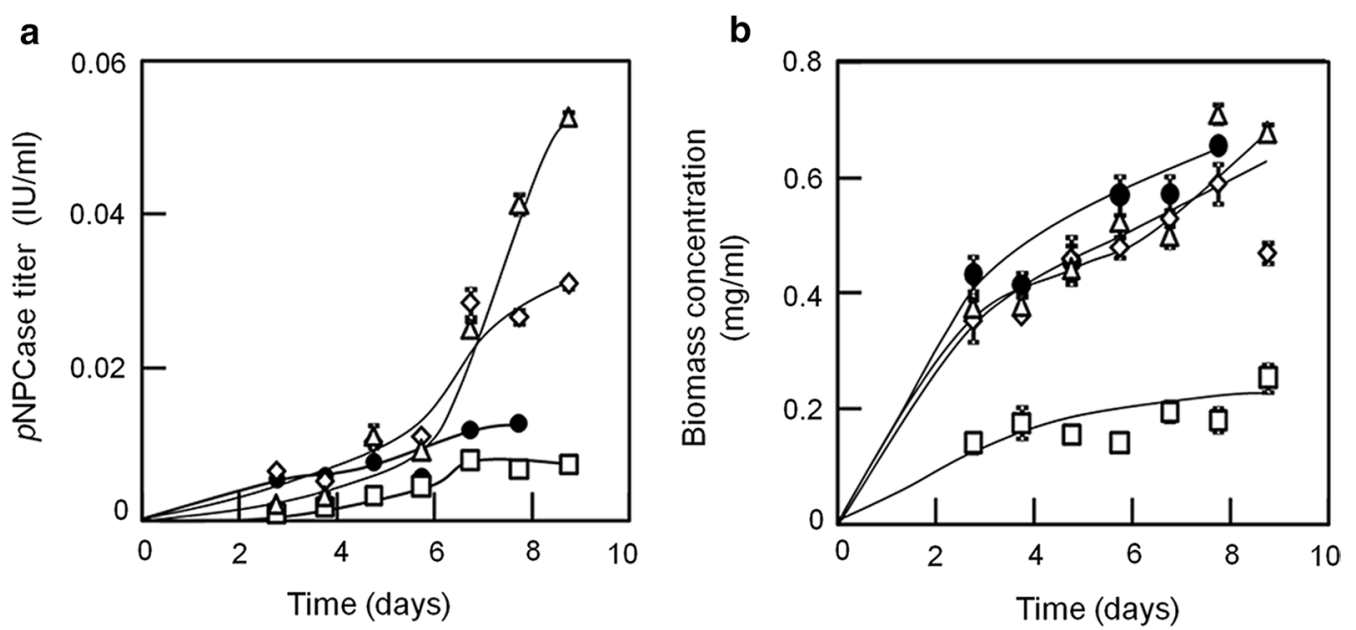

Fig. 6 Comparison of pNPCase titer and biomass accumulation among strains. The black sphere represents the parent strain, a white triangle represents Kuace2, a white diamond represents Kuxyr1, and a white square represents $\Delta c r e 1$. a $p N P C a s e$ titer; $\mathbf{b}$ biomass concentration

and 6.1- and 3.9-fold higher, respectively, than that of $\Delta c r e 1$ strain. To summarize, these results indicated that Kuace 2 and Kuxyr1 produced much higher $p$ NPCase titers than the parent and $\Delta c r e 1$ strains, and maintained a similar growth profile to that of the parent strain.

\section{Discussion}

Designing new strategies is important for the production of protein or other biochemicals in fungal species. Recent studies to increase cellulase production have primarily focused on the manipulation of Cre1/Mig1 (a yeast Cre1 homolog), for example, the use of gene knockouts or replacement of the gene with a non-functional allele [11, $20,41,42]$. However, this can affect biomass accumulation, which results in a decreased final product titer. Lactose is an economically feasible soluble carbon source for inducing cellulase production in T. reesei [34-37], which has been widely used in the enzyme industry [43-45]. However, an extracellular $\beta$-galactosidase (bga1) produced by $T$. reesei hydrolyzes lactose into $\mathrm{D}$-galactose and D-glucose, and bgal overexpression abolishes the expression of the two prominent cellulase-encoding genes cbh1 and cbh2 in T. reesei [46]. Therefore, CCR can be triggered when using lactose as a carbon source. In addition to $c e l 7 a$, we also observed that the expression of the cellulase genes such as cel6a, cel5a, and cel7b in T. reesei was significantly elevated in all fTF-containing transformants cultured on plates containing lactose [38] (Additional file 7: Figure S7). Recent studies have reported that an increased cellulolytic activity occurred as a result of the introduction of a truncated form of cre1, cre1-96, into T. reesei $[19,20]$. Although this approach is different from the one described in this study, the overexpression of either cre1-96 or fTF has no significant effect on $T$. reesei growth and exerts a positive effect on cellulase production by attenuating the effect of CCR.

Xyr1 is a major activator of cellulase production in $T$. reesei $[32,47]$. However, lactose-induced xyr1 expression requires the presence of Cre1 [48]. Therefore, despite mediating CCR, Cre1 also exerts positive effects on cellulase transcription under inducing conditions. Genome-wide analysis has revealed that Cre1 participates in several morphological events in $T$. reesei, such as hyphal development and sporulation [18]. The deletion of another $T$. reesei cellulase repressor, Ace1, also severely impairs fungal growth [39]. Therefore, the deletion of cre 1 or ace1 is not a good strategy to improve cellulase production in $T$. reesei.

The $T$. reesei cel7a gene has been proposed to be repressed via a Cre1 and Ace1-mediated double lock mechanism: Cre1 or Ace1 directly binds to the promoter region of $c e l 7 a$, followed by the repression of Xyr 1 expression by Cre1 and Ace1 [11, 32, 39, 47-49]. Therefore, one possible reason for the fTF-mediated elevation of cel7a expression is that fTFs can disturb the Cre1-and Ace1-mediated double lock by competing for Cre1- and Ace1-binding sites after the initiation of CCR during cellulase production. This hypothetical working mechanism is illustrated in Fig. 7.

Although the four fTFs possess the same zinc finger motifs, the cel7a transcription level was higher in strains containing Sace2 or Sxyr1 than in those containing Sace3 or Sclr2. The possible reasons for this phenomenon include the following: (i) a heterodimer composed of Xyr1 and Ace2 has been demonstrated to function as an activator of cellulase expression; (ii) 


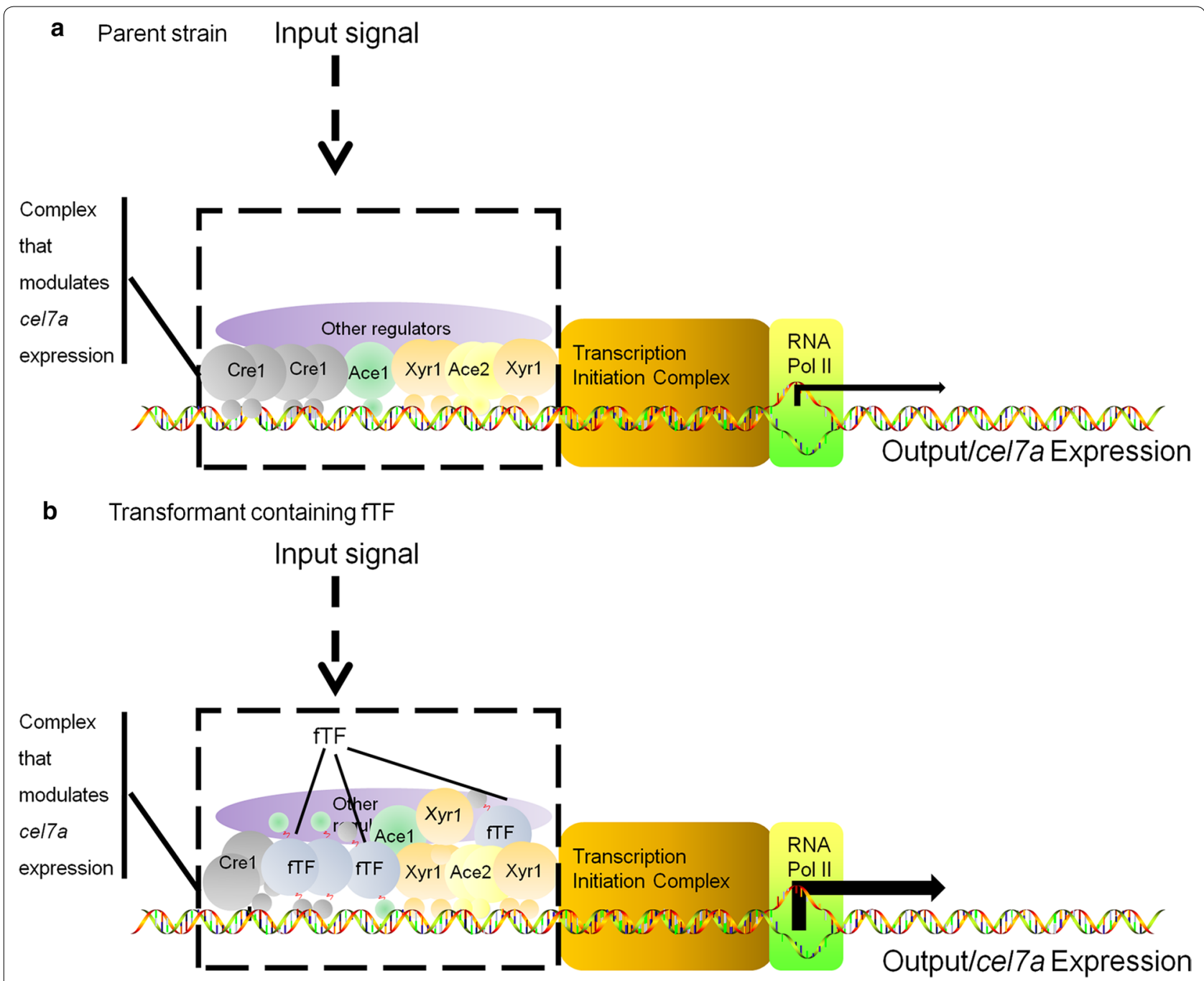

Fig. 7 Schematic diagrams showing cel7a transcriptional regulation in the parent and transformant strains. a parent strain; $\mathbf{b}$ transformants (Kuace3, Kuclr2, Kuace2, or Kuxyr1)

a Xyr1 homodimer has been implicated in the formation of a cellulase induction-specific complex [50,51]; (iii) there is no report on a homodimer or heterodimer composed of Ace3 or Clr2 found in the cellulase promoter regions in $T$. reesei; and (iv) it is possible that Sace 2 and Sxyr 1 may form more hetero- or homodimers with Xyr1 at the cel7a promoter region than with Sace 3 or Sclr2. Future studies will involve the introduction of the four fTFs (Sace3, Sclr2, Sace2, and Sxyr1) into T. reesei $\Delta x y r 1$ (tku70::hph; xyr1::pyr4) or T. reesei $\triangle a c e 2$ (tku70::hph; ace2::pyr4). The transcriptional levels of cellulase genes will be compared among all the transformants to investigate the relationship between fTFs and homodimer/heterodimer formation. These will be helpful for further understanding the role of the Xyr 1 and Ace 2 activation domains on cellulase transcription.
Synthetic or fusion transcription factors have been prepared for exploring the transcriptional regulation network and phenotypic engineering in yeast and mammalian cells [21, 22]. However, these studies do not address the delicate balance between product titer and strain growth. In this study, we designed four fusion or synthetic transcription factors in T. reesei, and found that two fTFs, Sace 2 and Sxyr 1, significantly improve the expression of a major cellulase gene without decreasing biomass accumulation and resulted in significantly enhanced cellulase activities. It is possible that the strategies described in this study could be applied in other fungal species, as phenomena related to Cre1-mediated CCR have been observed. For example, in the oleaginous yeast Yarrowia lipolytica, the growth rate and biomass accumulation of $\Delta m i g 1$ (a homologous gene of cre1) were found to be lower than those of the wild type strain; 
however, a 1.35-fold lipid increase in lipid content was observed [41].

\section{Conclusions}

Here, we developed a new strategy for increasing T. reesei cellulase titers. Four fTFs (Sace3, Sclr2, Sace2, and Sxyr1) were designed to release or attenuate the CCR inhibition of cellulase genes, and Cre1 was left intact to maintain strain growth. The Kuace3, Kuclr2, Kuace2, and Kuxyr1 strains were produced by transforming Sace3, Sclr2, Sace2, and Sxyr1, respectively, into the T. reesei genome. The growth of these transformants was roughly equivalent to that of the parent strain. However, the transcription levels of cel7a, which encodes the major cellulase protein cellobiohydrolase 1 , were significantly elevated in all transformants, particularly those in Kuace2 and Kuxyr1, when lactose was used as the carbon source. This suggests that a complete or partial release from CCR occurred. Furthermore, the use of fed-batch fermentation resulted in a significant increase in the $p$ NPCase titers of the Kuace 2 or Kuxyr1 strains compared with those of the parent and $\Delta$ cre 1 strains.

To summarize, a new strategy based on fTFs was successfully established to improve cellulase titers in T. ree$s e i$. In addition, this study provides valuable information for designing new fTFs for biochemical compounds production in other fungal species.

\section{Methods}

\section{Strains and reagents}

Trichoderma reesei $\Delta t k u 70$ (ATCC MYA-256), a nonhomologous end joining pathway-deficient strain, was used as the parent strain in this study [52]. All the other strains constructed in this study are listed in Additional file 8: Table S1. The E. coli strain GB05-dir was used for constructing all the plasmids [53].

Wheat bran was kindly provided by Longlive BioTechnology Co., Ltd. (Yucheng, Shandong, China). The $p$-nitrophenyl- $\beta$-D-cellobioside $(p \mathrm{NPC})$ was purchased from Sigma-Aldrich (St. Louis, MO, USA). Uridine, PEG6000, sorbitol, and lactose were purchased from Sangon Biotech Co., Ltd. (Shanghai, China). KOD FX DNA polymerase (Toyobo Co., Ltd., Osaka, Japan) was used for all polymerase chain reaction amplifications. RNAiso ${ }^{\text {тм }}$ reagent, PrimeScript ${ }^{\circledR}$ RT reagent Kit With gDNA Eraser (Perfect Real Time) and SYBR ${ }^{\circledR}$ Premix Ex Tag ${ }^{\mathrm{TM}}$ (Tli RNase H Plus) were purchased from Takara Bio Inc. (Shiga, Japan). The DIG High Prime DNA Labeling and Detection Starter Kit I (Roche Diagnostics, Mannheim, Germany) was used for Southern blotting analysis. The restriction enzymes Ase I and Xho I used for genome digestion were purchased from Thermo Fisher Scientific Inc. (Waltham, MA, USA). All other chemicals were purchased from Sinopharm Chemical Reagent Co., Ltd. (Shanghai, China).

\section{Construction of fTF replacement cassettes and the cre 1 deletion cassette}

All plasmids were constructed by linear-linear homologous recombination, which was mediated by full-length RecET in E. coli GB05-dir [53, 54]. The construction and composition of the pSxyr1 plasmid containing the Sxyr1 replacement cassette are as follows: $2 \mathrm{~kb}$ of sequence upand downstream of the $k u 70$ gene, the promoter and zinc finger regions of cre1 (comprising nucleotides -1364 to $417 \mathrm{bp}$ ), the activation domain of $x y r 1$ (445 to $2947 \mathrm{bp}$ ), and the zinc finger region of ace1 (1276 to $1716 \mathrm{bp}$ ) were each amplified from the $T$. reesei genome. A GSGGSGTS peptide was then used to link each domain. The pyr4 selective marker was obtained as previously described [55], and the $\operatorname{trp} C$ terminator was cloned from pSilent-1. The pUG6 plasmid was used as the accepter plasmid. A similar strategy was used to construct the pSace3, pSclr2, and pSace 2 plasmids. The activation domains of ace3 (comprising nucleotides 220-2035 bp), clr2 (100$2003 \mathrm{bp})$, and ace2 (118-1023 bp) were inserted into pSace3, pSclr2, and pSace2, respectively. A schematic diagram of the Sace3, Sclr2, Sace2, and Sxyr1 replacement cassettes is shown in Additional file 1: Figure S1A. The composition of the pCre1 plasmid containing the cre 1 deletion cassette included the $2 \mathrm{~kb}$ up- and downstream of the $k u 70$ gene, the pyr4 selective marker, and the pUG6 plasmid. A schematic diagram of the cre 1 deletion cassette is shown in Additional file 2: Figure S2A.

\section{Strain construction}

The transformation of Sace3, Sclr2, Sace2, and Sxyr1 replacement cassettes and the cre1 deletion cassette into $T$. reesei $\Delta t k u 70$ was performed using previously described methods [52]. The transformants were cultured on plates containing minimal medium $(\mathrm{MM})+2 \%$ glucose. The composition of MM is described in the literature [55]. Following two rounds of single-spore isolations, correct transformants with homologous integration were confirmed using PCR and Southern blotting.

\section{Growth and sporulation assays}

Approximately $1 \mathrm{~cm}^{3}$ of agar containing parent or mutant mycelia was inoculated on a solid plate containing PDA, wheat bran, MM with $2 \%$ glucose, or MM with $2 \%$ lactose. All media used for T. reesei $\Delta t k u 70$ culture were supplemented with $0.001 \%$ uridine. Plates were incubated at $30{ }^{\circ} \mathrm{C}$ for 6 days, and colony diameters were measured from the 2nd day. All experiments were performed in triplicates. Microscopic observation of hyphal morphology was based on a previously described method [56]. 
Approximately, $1 \times 10^{3}$ spores were inoculated on coverslips with solidified medium containing PDA, wheat bran, $\mathrm{MM}$ with $2 \%$ glucose, or MM with $2 \%$ lactose at $30{ }^{\circ} \mathrm{C}$ for $48 \mathrm{~h}$. Microscopic images of hyphae (Nikon Eclipse E100, 400 $\times$ magnification) were then captured using a Nikon D5000 camera.

Approximately, $1 \times 10^{6}$ spores of parent strain and transformants were inoculated on PDA and wheat bran plates and incubated at $30{ }^{\circ} \mathrm{C}$ for 6 days. Spores were then harvested from the plates using a solution containing $0.9 \% \mathrm{NaCl}$ and $0.5 \%$ Tween- 80 and enumerated with a hemocytometer. Colony diameters were measured on the 6 th day of incubation. The number of spores produced was normalized to the colony area.

\section{Identification of fTFs that improve cellulase transcription in $T$. reesei}

Approximately, $1 \times 10^{7}$ spores of the parent strain and transformants containing fTFs were inoculated into liquid medium containing $\mathrm{MM}+2 \%$ glucose $+0.001 \%$ tryptone and cultured at $30{ }^{\circ} \mathrm{C}$ and $200 \mathrm{rpm}$ for $48 \mathrm{~h}$. The mycelia were filtered and washed with MM solution. Equal amounts of mycelia were transferred into submerged medium containing $\mathrm{MM}+105 \mathrm{mM}$ glycerol and the samples were incubated at $30{ }^{\circ} \mathrm{C}$ and $200 \mathrm{rpm}$ for $24 \mathrm{~h}$. The mycelia were harvested, and approximately $0.1 \mathrm{~g}$ of harvested mycelia was transferred to $100 \mathrm{~mL}$ liquid medium containing $\mathrm{MM}+2 \mathrm{~g}$ lactose. The mycelia were cultured at $30{ }^{\circ} \mathrm{C}$ and $200 \mathrm{rpm}$. Samples were taken at $1,6,8$, and $12 \mathrm{~h}$ after inoculation and immediately frozen at $-80{ }^{\circ} \mathrm{C}$. These samples were used for determining the transcriptional levels of cellulase transcription factors and cellulase genes.

The extraction of total RNA and subsequent synthesis of cDNA were performed as previously described [57]. Real-time PCR reactions were performed using a LightCycler 96 Real-Time PCR system (Roche Applied Science, Mannheim, Germany). The real-time PCR reactions were performed according to the manufacturer's instructions for SYBR Premix Ex Taq ${ }^{\text {TM }}$ (Ti RNaseH Plus) (Takara Bio Inc., Shiga, Japan). The relative transcription levels of cellulase genes and transcription factors were calculated using the $2^{-\triangle \Delta C T}$ method [58]. The primers used for realtime PCR are listed in Additional file 9: Table S2.

\section{Assessment of $p$ NPCase activity}

To determine $p$ NPCase activities, mycelia were precultured as described above. Approximately $0.2 \mathrm{~g}$ of mycelia was inoculated in $200 \mathrm{~mL}$ submerged medium containing $\mathrm{MM}$ solution $+4 \mathrm{~g}$ lactose and incubated at $30{ }^{\circ} \mathrm{C}$ and $200 \mathrm{rpm}$. Culture samples $(30 \mathrm{~mL})$ were collected on days 4,5 , and 6 after inoculation and centrifuged at 12,000 rpm for $10 \mathrm{~min}$. The solid sediment was extracted and dried at $100{ }^{\circ} \mathrm{C}$ for $24 \mathrm{~h}$ [39]. The supernatant $(25 \mathrm{~mL})$ was extracted and concentrated to a final volume of $1 \mathrm{~mL}$ using Vivaspin 15R centrifugal devices with a $3 \mathrm{kDa}$ molecular weight cutoff (Sartorius, Goettingen, Germany). The concentrated supernatant was then mixed with $20 \mathrm{~mL}$ of $50 \mathrm{mM}$ acetic acid buffer ( $\mathrm{pH} 4.8)$ and concentrated again to a final volume of $1 \mathrm{~mL}$. The $p$ NPCase activity was measured as previously described [55] and normalized using the dry mycelium weight.

\section{Cellulase production using a fed-batch culture in a 3-L fermenter}

Trichoderma reesei was cultured using a protocol based on a previously published method [43]. Briefly, samples of the parent and mutant strains (approximately $1 \times 10^{7}$ spores) were inoculated in $100 \mathrm{~mL}$ liquid medium containing MM solution $+2 \%$ glucose $+0.001 \%$ tryptone and incubated at $30{ }^{\circ} \mathrm{C}$ and $200 \mathrm{rpm}$. After $48 \mathrm{~h}$ of cultivation, the samples were used as seed cultures for fermentation. The seed culture $(200 \mathrm{~mL})$ was used to inoculate a liquid medium containing $1.8 \mathrm{~L}$ of MM solution, 6 g glucose, $10 \mathrm{~g}$ Avicel, and $2 \mathrm{~g}$ tryptone in a 3-L fermenter (Model BLB10-3GJG, Bailun Bio-Technology Co., Ltd., Shanghai, China). The feeding solutions contained $400 \mathrm{~mL}$ of carbon source $(150 \mathrm{~g}$ lactose $/ \mathrm{L})$ and $400 \mathrm{~mL}$ of nitrogen source $\left(160 \mathrm{~g}\left(\mathrm{NH}_{4}\right)_{2} \mathrm{SO}_{4} / \mathrm{L}\right)$. The initial $\mathrm{pH}$ value was regulated at $\mathrm{pH} 4.5$ and maintained at $\mathrm{pH} 4.0-5.0$ by the automatic addition of $3 \mathrm{M} \mathrm{H}_{3} \mathrm{PO}_{4}$ and $3 \mathrm{M} \mathrm{KOH}$ during the entire fermentation process. The airflow was $1.5 \mathrm{~L} /$ min and the agitation rate was adjusted to maintain the dissolved oxygen level above $30 \%$. The media were fed following $66 \mathrm{~h}$ of cultivation. The injection rates of the carbon and nitrogen solutions were $0.72 \mathrm{~g} / \mathrm{h}$ and $0.23 \mathrm{~g} / \mathrm{h}$, respectively. Samples were periodically collected to measure the $p$ NPCase titer and intracellular protein concentration. The measurements of intracellular protein concentration to determine the biomass concentration were performed according to a previously described method [56].

\section{Statistical analysis}

All experiments were performed in triplicates. Statistical analyses were performed using two-tailed Student's $t$ tests, and $p$ values of $<0.05$ were considered statistically significant.

\section{Supplementary information}

\section{Supplementary information accompanies this paper at https://doi. org/10.1186/s13068-019-1589-2.}

Additional file 1: Figure S1. Southern blotting analysis of parent and fTF-containing transformants. A) Schematic diagram of Southern blotting. B) Southern blot of the parent, Kuace3, and Kuclr2 strains. C) Southern blot of the parent, Kuace2, and Kuxyr1 strains. 1: 2 kb upstream of ku70; 2: cre 1 
promoter; 3: Sace3; 4: trpC terminator; 5: pyr4; 6: 2 kb downstream of ku70; 7: Sclr2; 8: Sace2; 9: Sxyr1. Lane M: 1 kb molecular weight marker, Lane 1: parent strain, Lane 2: Kuace3 strain, Lane 3: Kuclr2 strain, Lane 4: Kuace2 strain, Lane 5: Kuxyr1 strain. The arrows indicate the predicted size of each band.

Additional file 2: Figure S2. Southern blotting analysis of the $\Delta$ cre 1 strain. A) Schematic diagram of Southern blotting. B) Southern blot of parent and $\Delta$ crel strains. 1:2 kb upstream of crel gene; $2:$ pyr $4 ; 3: 2 \mathrm{~kb}$ downstream of cre1. Lane M: 1 kb molecular weight marker, Lane 1: parent strain, Lane 2: $\Delta c r e 1$ strain. The arrows indicate the predicted size of each strain.

Additional file 3: Figure S3. Colony diameters of parent strain and transformants on plates containing different carbon sources. Black represents cultivation for 2 days, white represents cultivation for 3 days, dark gray represents cultivation for 4 days, and light gray represents cultivation for 5 days.

Additional file 4: Figure S4. Microscopic observation of the hyphae of the parent strain and transformants. Scale: $100 \mu \mathrm{m}$.

Additional file 5: Figure S5. Sporulation of the parent strain and transformants. Black represents wheat bran as the culture medium; white represents PDA as the culture medium.

Additional file 6: Figure S6. Transcriptional levels of fTFs among the transformants. The transcriptional level of fTFs was normalized to that of actin. Dark gray represents Kuace3, white represents Kuclr2, black represents Kuace2, and light gray represents Kuxyr1.

Additional file 7: Figure S7. Transcriptional levels of cel6a, cel5a, and cel7b among the transformants. The transcriptional levels of cel6a, cel5a, and cel/b were normalized to that of actin (data presented are mean \pm SEM; ${ }^{*} p<0.05,{ }^{* *} p<0.01,{ }^{* *} p<0.001, \mathrm{n}=3$; two-tailed Student's $t$ tests). A left slash represents the parent strain, dark gray represents Kuace3, white represents Kuclr2, black represents Kuace2, and light gray represents Kuxyr1.

Additional file 8: Table S1. T. reesei strains used in this study.

Additional file 9: Table S2. Primers used for real-time PCR.

\section{Abbreviations}

CCR: Carbon catabolite repression; fTF: Fusion transcription factor; $p N P C$ : p-Nitrophenyl- $\beta$-D-cellobioside; MM: Minimal medium; PDA: Potato dextrose agar.

\section{Acknowledgements}

We thank Ms Shaoli Hou for technical help and Prof. Dr. Weiwen Zhang for polishing the English language.

\section{Authors' contributions}

$X F$ and $F Z W$ conceived the project and designed the experiments. FZW, RQZ, WG, KLN, and LJH performed the experiments. FZW and XF analyzed the data and wrote the manuscript. ZQD, YCL, CJJ and XF revised the manuscript. All the authors discussed the manuscript and agreed to its publication. All authors read and approved the final manuscript.

\section{Funding}

This work was supported by the National Key Research and Development Program (No. 2018YFB1501701), Key Technologies R\&D Program of Shandong Province (No. 2018GSF121021), the Tianjin Municipal Science Foundation (No. 18JCQNJC10000), the State Key Laboratory of Microbial Technology Open Projects Fund and National Natural Science Foundation of China (No. 31570040 and 31870785).

\section{Availability of data and materials}

All data generated or analyzed during this study are included in this published article and the additional files.

Ethics approval and consent to participate Not applicable.

\section{Consent for publication}

All authors agreed to the publication of this article.

\section{Competing interests}

The authors declare that they have no competing interests.

\section{Author details}

1 State Key Laboratory of Microbial Technology, Shandong University, Qingdao 266237, China. ${ }^{2}$ Center for Biosafety Research and Strategy, Tianjin University, Tianjin, China. ${ }^{3}$ School of Chemistry and Chemical Engineering, Shandong University, Jinan, Shandong, China.

Received: 14 May 2019 Accepted: 9 October 2019

Published online: 15 October 2019

\section{References}

1. Rubin EM. Genomics of cellulosic biofuels. Nature. 2008;454:841-5.

2. Kubicek CP, Mikus M, Schuster A, Schmoll M, Seiboth B. Metabolic engineering strategies for the improvement of cellulase production by Hypocrea jecorina. Biotechnol Biofuels. 2009;2:19.

3. Unrean P. Bioprocess modelling for the design and optimization of lignocellulosic biomass fermentation. Bioresour Bioprocess. 2016;3:1.

4. Yang X, Ma X, Li H, Chen J, Fang S. Effects of hot-washing process on structure and enzymatic hydrolysis of treated steam explosion corn stover. Bioresour Bioprocess. 2016;3:39.

5. Fujii T, Fang X, Inoue H, Murakami K, Sawayama S. Enzymatic hydrolyzing performance of Acremonium cellulolyticus and Trichoderma reesei against three lignocellulosic materials. Biotechnol Biofuels. 2009;2:24.

6. Ire FS, Ezebuiro V, Ogugbue CJ. Production of bioethanol by bacterial co-culture from agro-waste-impacted soil through simultaneous saccharification and co-fermentation of steam-exploded bagasse. Bioresour Bioprocess. 2016;3:26.

7. Merino ST, Cherry J. Progress and challenges in enzyme development for biomass utilization. Adv Biochem Eng Biotechnol. 2007;108:95-120.

8. Klein-Marcuschamer D, Oleskowicz-Popiel P, Simmons BA, Blanch HW. The challenge of enzyme cost in the production of lignocellulosic biofuels. Biotechnol Bioeng. 2012;109:1083-7.

9. Sassner P, Galbe M, Zacchi G. Techno-economic evaluation of bioethanol production from three different lignocellulosic materials. Biomass Bioenerg. 2008;32:422-30.

10. Seiboth B, Herold S, Kubicek CP. Metabolic engineering of inducer formation for cellulase and hemicellulase gene expression in Trichoderma reesei. Subcell Biochem. 2012;64:367-90.

11. Gupta VK, Steindorff AS, de Paula RG, Silva-Rocha R, Mach-Aigner AR, Mach RL, Silva RN. The post-genomic era of Trichoderma reesei: what's Next? Trends Biotechnol. 2016;34:970-82.

12. Derntl C, Gudynaite-Savitch L, Calixte S, White T, Mach RL, Mach-Aigner AR. Mutation of the xylanase regulator 1 causes a glucose blind hydrolase expressing phenotype in industrially used Trichoderma strains. Biotechnol Biofuels. 2013;6:62.

13. Liu GD, Zhang L, Qin YQ, Zou G, Li ZH, Yan X, Wei XM, Chen M, Chen L, Zheng K, Zhang J, Ma L, Li J, Liu R, Xu H, Bao XM, Fang X, Wang LS, Zhong YH, Liu WF, Zheng HJ, Wang SY, Wang CS, Xun LY, Zhao GP, Wang TH, Zhou ZH, Qu YB. Long-term strain improvements accumulate mutations in regulatory elements responsible for hyper-production of cellulolytic enzymes. Sci Rep. 2013:3:1569.

14. Yao GS, Li ZH, Gao LW, Wu RM, Kan QB, Liu GD, Qu YB. Redesigning the regulatory pathway to enhance cellulase production in Penicillium oxalicum. Biotechnol Biofuels. 2015;8:71.

15. Long C, Cheng Y, Cui J, Liu J, Gan L, Zeng B, Long M. Enhancing cellulase and hemicellulase production in Trichoderma orientalis EU7-22 via knockout of the creA. Mol Biotechnol. 2017;60:55-61.

16. Sun J, Glass NL. Identification of the CRE-1 cellulolytic regulon in Neurospora crassa. PLoS ONE. 2011;6:e25654.

17. Nakari-Setälä T, Paloheimo M, Kallio J, Vehmaanperä J, Penttilä M, Saloheimo M. Genetic modification of carbon catabolite repression in Trichoderma reesei for improved protein production. Appl Environ Microbiol. 2009;75:4853-60 
18. Portnoy T, Margeot A, Linke R, Atanasova L, Fekete E, Sándor E, Hartl L, Karaffa L, Druzhinina IS, Seiboth B, Le Crom S, Kubicek CP. The CRE1 carbon catabolite repressor of the fungus Trichoderma reesei: a master regulator of carbon assimilation. BMC Genomics. 2011;12:269.

19. Mello-de-Sousa TM, Gorsche R, Rassinger A, Pocas-Fonseca MJ, Mach RL, Mach-Aigner AR. A truncated form of the carbon catabolite repressor 1 increases cellulase production in Trichoderma reesei. Biotechnol Biofuels. 2014;7:129.

20. Rassinger A, Gacek-Matthews A, Strauss J, Mach RL, Mach-Aigner AR. Truncation of the transcriptional repressor protein $\mathrm{Cre} 1$ in Trichoderma reesei Rut-C30 turns it into an activator. Fungal Biol Biotechnol. 2018;5:15.

21. Khalil AS, LuTK, Bashor CJ, Ramirez CL, Pyenson NC, Joung JK, Collins JJ. A synthetic biology framework for programming eukaryotic transcription functions. Cell. 2012;150:647-58.

22. Park KS, Lee DK, Lee H, Lee Y, Jang YS, Kim YH, Yang HY, Lee SI, Seol W, Kim JS. Phenotypic alteration of eukaryotic cells using randomized libraries of artificial transcription factors. Nat Biotechnol. 2003;21:1208-14.

23. Hou JR, Zeng WQ, Zong YQ, Chen ZH, Miao CS, Wang BJ, Lou CB. Engineering the ultrasensitive transcription factors by fusing a modular oligomerization domain. Acs Synth Biol. 2018;7:1188-94.

24. Naseri G, Balazadeh S, Machens F, Kamranfar I, Messerschmidt K, MuellerRoeber B. Plant-derived transcription factors for orthologous regulation of gene expression in the yeast Saccharomyces cerevisiae. Acs Synth Biol. 2017:6:1742-56.

25. Peterson R, Nevalainen $\mathrm{H}$. Trichoderma reesei RUT-C30-thirty years of strain improvement. Microbiol-Sgm. 2012;158:58-68.

26. Liu K, Lin XH, Yue J, Li XZ, Fang X, Zhu MT, Lin JQ, Qu YB, Xiao L. High concentration ethanol production from corncob residues by fed-batch strategy. Bioresour Technol. 2010;101:4952-8.

27. Fang $X$, Yano S, Inoue H, Sawayama S. Strain improvement of Acremonium cellulolyticus for cellulase production by mutation. J Biosci Bioeng. 2009;107:256-61.

28. Tian CG, Beeson WT, lavarone AT, Sun JP, Marletta MA, Cate JHD, Glass NL. Systems analysis of plant cell wall degradation by the model filamentous fungus Neurospora crassa. Proc Natl Acad Sci USA. 2009;106:22157-62.

29. Martinez D, Berka RM, Henrissat B, Saloheimo M, Arvas M, Baker SE, Chapman J, Chertkov O, Coutinho PM, Cullen D, Danchin EG, Grigoriev IV, Harris P, Jackson M, Kubicek CP, Han CS, Ho I, Larrondo LF, de Leon AL, Magnuson JK, Merino S, Misra M, Nelson B, Putnam N, Robbertse B, Salamov AA, Schmoll M, Terry A, Thayer N, Westerholm-Parvinen A, Schoch CL, Yao J, Barabote R, Nelson MA, Detter C, Bruce D, Kuske CR, Xie G, Richardson P, Rokhsar DS, Lucas SM, Rubin EM, Dunn-Coleman N, Ward M, Brettin TS. Genome sequencing and analysis of the biomass-degrading fungus Trichoderma reesei (syn. Hypocrea jecorina). Nat Biotechnol. 2008;26:553-60.

30. Saloheimo M, Pakula TM. The cargo and the transport system: secreted proteins and protein secretion in Trichoderma reesei (Hypocrea jecorina). Microbiol-Sgm. 2012;158:46-57.

31. Esterbauer H, Steiner W, Labudova I, Hermann A, Hayn M. Production of Trichoderma cellulase in laboratory and pilot scale. Bioresour Technol. 1991;36:51-65.

32. Bischof RH, Ramoni J, Seiboth B. Cellulases and beyond: the first 70 years of the enzyme producer Trichoderma reesei. Microb Cell Fact. 2016;15:106.

33. Craig JP, Coradetti ST, Starr TL, Glass NL. Direct target network of the Neurospora crassa plant cell wall deconstruction regulators CLR-1, CLR-2, and XLR-1. Mbio. 2015;6:e01452-15.

34. Ivanova C, Bååth JA, Seiboth B, Kubicek CP. Systems analysis of lactose metabolism in Trichoderma reesei identifies a lactose permease that is essential for cellulase induction. PLoS ONE. 2013;8:e62631.

35. Morikawa Y, Ohashi T, Mantani O, Okada H. Cellulase induction by lactose in Trichoderma reesei PC-3-7. Appl Microbiol Biotechnol. 1995;44:106-11.

36. Wen Z, Liao W, Chen S. Production of cellulase by Trichoderma reesei from dairy manure. Bioresour Technol. 2005;96:491-9.

37. Bailey MJ, Tähtiharju J. Efficient cellulase production by Trichoderma reesei in continuous cultivation on lactose medium with a computer-controlled feeding strategy. Appl Microbiol Biotechnol. 2003;62:156-62.

38. Herpoël-Gimbert I, Margeot A, Dolla A, Jan G, Mollé D, Lignon S, Mathis H, Sigoillot JC, Monot F, Asther M. Comparative secretome analyses of two Trichoderma reesei RUT-C30 and CL847 hypersecretory strains. Biotechnol Biofuels. 2008;1:18.

39. Aro N, Ilmén M, Saloheimo A, Penttilä M. ACEl of Trichoderma reesei is a repressor of cellulase and xylanase expression. Appl Environ Microbiol. 2003;69:56-65.
40. Bischof R, Fourtis L, Limbeck A, Gamauf C, Seiboth B, Kubicek CP. Comparative analysis of the Trichoderma reesei transcriptome during growth on the cellulase inducing substrates wheat straw and lactose. Biotechnol Biofuels. 2013;6:127.

41. Wang ZP, Xu HM, Wang GY, Chi Z, Chi ZM. Disruption of the MIG1 gene enhances lipid biosynthesis in the oleaginous yeast Yarrowia lipolytica ACADC 50109. Bba-Mol Cell Biol L. 2013;1831:675-82.

42. Li ZH, Yao GS, Wu RM, Gao LW, Kan QB, Liu M, Yang P, Liu GD, Qin YQ, Song $X$, Zhong YH, Fang X, Qu YB. Synergistic and dose-controlled regulation of cellulase gene expression in Penicillium oxalicum. PLoS Genet. 2015;11:e1005509.

43. Ahamed A, Vermette P. Culture-based strategies to enhance cellulase enzyme production from Trichoderma reesei RUT-C30 in bioreactor culture conditions. Biochem Eng J. 2008;40:399-407.

44. Sehnem NT, De Bittencourt LR, Camassola M, Dillon AJP. Cellulase production by Penicillium echinulatum on lactose. Appl Microbiol Biotechnol. 2006;72:163-7.

45. Fang X, Yano S, Inoue H, Sawayama S. Lactose enhances cellulase production by the filamentous fungus Acremonium cellulolyticus. J Biosci Bioeng. 2008;106:115-20.

46. Seiboth B, Hartl L, Salovuori N, Lanthaler K, Robson GD, Vehmaanperä J, Penttilä ME, Kubicek CP. Role of the bga1-encoded extracellular \{beta\}galactosidase of Hypocrea jecorina in cellulase induction by lactose. Appl Environ Microbiol. 2005;71:851-7.

47. Lichius A, Bidard F, Buchholz F, Le Crom S, Martin J, SchackwitzW, Austerlitz T, Grigoriev IV, Baker SE, Margeot A, Seiboth B, Kubicek CP. Genome sequencing of the Trichoderma reesei QM9136 mutant identifies a truncation of the transcriptional regulator XYR1 as the cause for its cellulase-negative phenotype. BMC Genomics. 2015;16:326.

48. Portnoy T, Margeot A, Seidl-Seiboth V, Le Crom S, Ben Chaabane F, Linke R, Seiboth B, Kubicek CP. Differential regulation of the cellulase transcription factors XYR1, ACE2, and ACE1 in Trichoderma reesei strains producing high and low levels of cellulase. Eukaryot Cell. 2011;10:262-71.

49. Mach-Aigner AR, Pucher ME, Steiger MG, Bauer GE, Preis SJ, Mach RL. Transcriptional regulation of $x y r 1$, encoding the main regulator of the xylanolytic and cellulolytic enzyme system in Hypocrea jecorina. Appl Environ Microbiol. 2008;74:6554-62.

50. Stricker AR, Mach RL, de Graaff $L H$. Regulation of transcription of cellulasesand hemicellulases-encoding genes in Aspergillus niger and Hypocrea jecorina (Trichoderma reesei). Appl Microbiol Biotechnol. 2008;78:211-20.

51. Stricker AR, Grosstessner-Hain K, Würleitner E, Mach RL. Xyr1 (xylanase regulator 1) regulates both the hydrolytic enzyme system and D-xylose metabolism in Hypocrea jecorina. Eukaryot Cell. 2006;5:2128-37.

52. Zhang GT, Hartl L, Schuster A, Polak S, Schmoll M, Wang TH, Seidl V, Seiboth B. Gene targeting in a nonhomologous end joining deficient Hypocrea jecorina. J Biotechnol. 2009;139:146-51.

53. Fu J, Bian X, Hu S, Wang H, Huang F, Seibert PM, Plaza A, Xia L, Müller R, Stewart AF, Zhang Y. Full-length RecE enhances linear-linear homologous recombination and facilitates direct cloning for bioprospecting. Nat Biotechnol. 2012;30:440-6.

54. Yin J, Hoffmann M, Bian X, Tu Q, Yan F, Xia L, Ding X, Stewart AF, Müller R, Fu $J$, Zhang Y. Direct cloning and heterologous expression of the salinomycin biosynthetic gene cluster from Streptomyces albus DSM41398 in Streptomyces coelicolor A3(2). Sci Rep. 2015;5:15081.

55. Wang MY, Zhao QS, Yang JH, Jiang BJ, Wang FZ, Liu KM, Fang X. A mitogenactivated protein kinase tmk3 participates in high osmolarity resistance, cell wall integrity maintenance and cellulase production regulation in Trichoderma reesei. PLoS ONE. 2013;8:e72189.

56. Wang FZ, Liu KM, Han L, Jiang BJ, Wang MY, Fang X. Function of a p24 heterodimer in morphogenesis and protein transport in Penicillium oxalicum. Sci Rep. 2015;5:11875.

57. Wang $F Z$, Liang $Y$, Wang MY, Yang H, Liu KM, Zhao Q, Fang X. Functional diversity of the p24gamma homologue Erp reveals physiological differences between two filamentous fungi. Fungal Genet Biol. 2013;61:15-22.

58. Livak KJ, Schmittgen TD. Analysis of relative gene expression data using real-time quantitative PCR and the 2(-Delta Delta C(T)) Method. Methods. 2001;25:402-8.

\section{Publisher's Note}

Springer Nature remains neutral with regard to jurisdictional claims in published maps and institutional affiliations. 